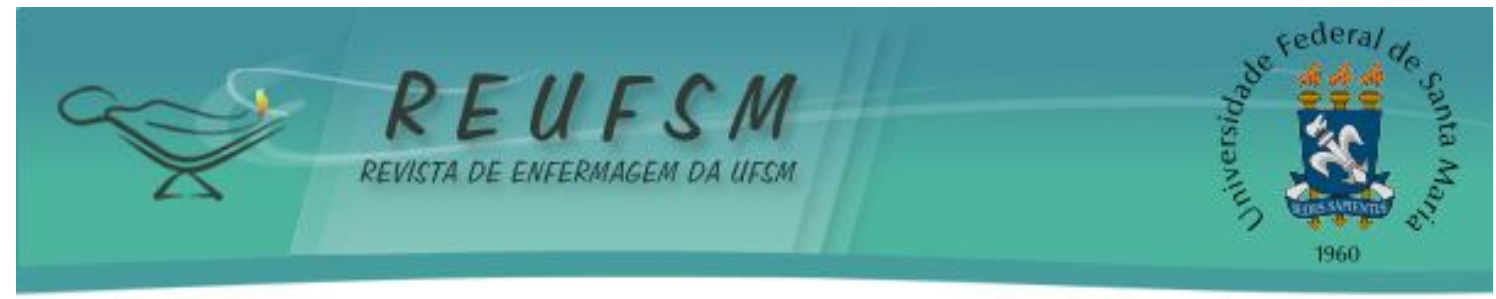

ARTIGO ORIGINAL

\title{
CONHECIMENTO DOS TRABALHADORES DA INDÙSTRIA METAL-MECÂNICA SOBRE FATORES DE RISCO PARA DOENÇA ARTERIAL CORONARIANA
}

\section{MECHANICAL METAL INDUST RY WORKER'S KNOWLEDGE ABOUT RISK FACTORS FOR CORONARY ARTERY DISEASE}

\section{CONOCIMIE NTO DE LOS TRABAJ ADORES DE LA INDUST RIA MET ALMECÁNICA SOBRE FACTORES DE RIESGO PARA ENFERMEDAD ARTERIAL CORONARIA}

\author{
Silvia Bohrer Oliva ${ }^{1}$ \\ Adriana Aparecida Paz ${ }^{2}$ \\ Emiliane Nogueira de Souza ${ }^{3}$
}

\begin{abstract}
RESUMO: Objetivo: identificar o conhecimento dos trabalhadores da indústria metalmecânica em relação aos fatores de risco para doença arterial coronariana (DAC) e às atitudes voltadas a sua prevenção. Métodos: estudo com delineamento transversal, realizado com um grupo de trabalhadores da indústria metal-mecânica do sul do Brasil. A amostra foi por conveniência. Os dados foram coletados por meio de um questionário e verificação de medidas antropométricas. Análise descritiva. Resultados: foram incluídos 100 trabalhadores, com idade média de 29,0+10,16 anos, $66 \%$ homens. A obesidade foi considerada como fator de risco para DAC por $94 \%$ dos trabalhadores, a hipertensão arterial por $88 \%$ e hipercolesterolemia por $80 \%$ Em relação às atitudes para prevenção da DAC, 51\% da amostra referiram praticar atividade física. Conclusão: a maioria dos entrevistados conhece os principais fatores de risco para DAC. Contudo, as atitudes voltadas à prevenção ainda são limitadas.
\end{abstract}

Descritores: Enfermagem; Enfermagem do trabalho; Saúde do trabalhador; Doença das coronárias; Fatores de risco.

ABSTRACT: Objective: Identify the worker's knowledge of metal industry mechanics in relation to risk factors for coronary artery disease (CAD) and attitudes aimed at prevention. Methods: A cross-sectional study, held with a group of employees of the metalworking industry from the south of Brazil. The sample was for convenience. Data were collected through a check questionnaire and anthropometric measures. Data analysis was descriptive. Results: 100 employees were included, with an average age of 29.0 +10.16 years, $66 \%$ men. Obesity was considered as a risk factor for CAD by $94 \%$ workers, hypertension by $88 \%$ and hypercholesterolemia by $80 \%$

Regarding the attitudes for CAD prevention, $51 \%$ of the sample reported physical activity. Conclusion: Most interviewees know the main risk factors for CAD. However, attitudes aimed at prevention are still limited.

Descriptors: Nursing; Occupational health nursing; Occupational health; Coronary disease; Risk factors.

${ }^{1}$ Enfermeira graduada pela Faculdade Enfermagem Nossa Senhora de Fátima (FÁTIMA). Caxias do Sul, RS, Brasil.

2 Enfermeira. Doutoranda pelo Programa de Pós-Graduação em Enfermagem da Universidade Federal do Rio Grande do Sul (UFRGS). Docente do Departamento de Enfermagem da Universidade Federal de Ciências da Saúde de Porto Alegre (UFCSPA) e do Curso de Enfermagem FÁTIMA. Membro do Grupo Interdisciplinar em Saúde Ocupacional (GISO/UFRGS). Porto Al egre, RS, Brasil. E-mail: adrianap@ufcspa.edu.br

3 Enfermeira. Doutoranda pelo Programa de Pós-Graduação em Ciências da Saúde: Cardiologia e Ciências Cardiovasculares da UFRGS. Docente do Departamento de Enfermagem da UFCSPA. Líder do Grupo de Estudo e Pesquisa sobre Práticas de Cuidado na Promoção da Saúde do Adulto com Alterações Cardiovasculares (PROCARDIO/IC-FUC). E-mail: emilianes@ufcspa.edu.br 


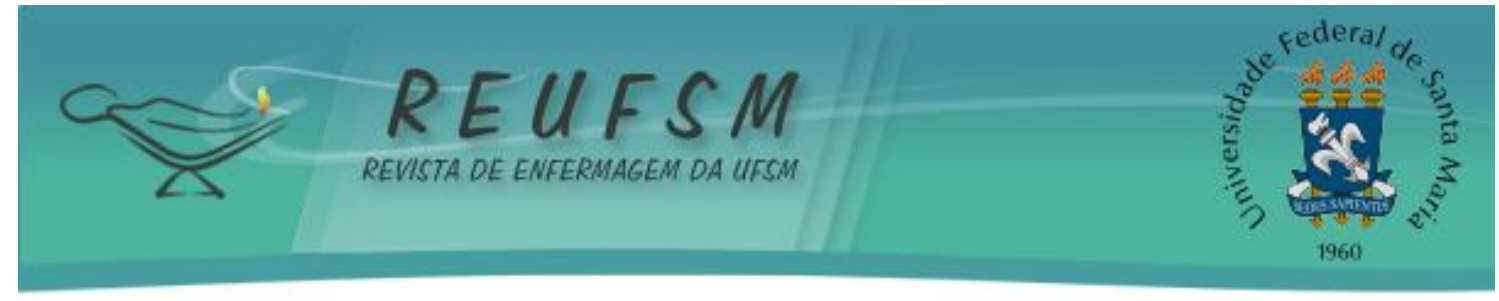

RESUMEN: Objetivo: identificar el conocimiento de los trabajadores de la industria metalmecánica en lo tocante a los factores de riesgo para la enfermedad arterial coronaria (EAC) y las actitudes dirigidas a la prevención. Métodos: estudio con delineamiento transversal, realizado con un grupo de trabajadores de la industria metalmecánica del sur de Brasil. El muestreo fue por conveniencia. Los datos se colectaron a través de un cuestionario y de la verificación de medidas antropométricas. Análisis descriptivo. Resultados: se incluyó a 100 trabajadores, con edades promedio de $29,0 \pm 10,16$ años, un $66 \%$ de hombres. La obesidad fue considerada como un factor de riesgo para EAC por un $94 \%$ de los trabajadores, la hipertensión arterial por un $88 \%$ y la hipercolesterolemia por un $80 \%$ Con relación a las actitudes para prevención de la EAC, un $51 \%$ del muestreo afirmó practicar actividad física. Conclusión: la mayoría de los entrevistados conoce los principales factores de riesgo para EAC. Sin embargo, las actitudes dirigidas a la prevención todavía son limitadas.

Descriptores: Enfermería; Enfermería del trabajo; Salud laboral; Enfermedad coronaria; Factores de risco.

\section{INTRODUÇÃO}

As doenças cardiovasculares (DCV) contribuem consideravelmente para a alta morbidade e mortalidade em países desenvolvidos e em desenvolvimento. 0 aumento da incidência de DCV exige intervenções que sejam eficazes, por isso, tem sido alvo de vários estudos e despertado o interesse por atingir grandes contingentes populacionais, além de representar elevados custos sociais e econômicos. ${ }^{1}$

No Brasil, em 2009, a mortalidade por doenças do aparelho circulatório foi o desfecho para o óbito de 318.798(29\%) brasileiros sendo, na sua maioria, do sexo masculino $(50,5 \%$. No Rio Grande do Sul (RS), a mortalidade atingiu 23.207(30,2\%) gaúchos, no mesmo período, sendo prevalente esta causa entre as mulheres (51\%). Em Caxias do Sul (RS), no mesmo período, o óbito ocorreu para 635(26,9\%) pessoas sendo, na sua maioria, do sexo feminino $(54,3 \%){ }^{2}$ A estimativa populacional no processo de envelhecimento implica no aumento da incidência e da prevalência de agravos de longa duração, entre elas as DCV.

Entre as DCV, destaca-se a doença arterial coronariana (DAC) que é considerada multifatorial, e a prevenção passa pela identificação do conjunto dos fatores de risco (FR). Os FR não modificáveis incluem a idade, o sexo e a história familiar positiva para DAC. Entre os modificáveis, estão a dislipidemia, o Diabetes Melito (DM), o tabagismo, 0 sedentarismo, a Hipertensão Arterial Sistêmica (HAS) e a obesidade. ${ }^{3}$ Destaca-se que a prevenção da DAC tem sido baseada no conhecimento dos FR modificáveis. Dados epidemiológicos mostram que a probabilidade de um indivíduo de 50 anos, sem exposição aos FR, venha desenvolver um evento coronariano, é de $6 \%$ em 10 anos. Além disso, 0 conhecimento dos FR permite que se elaborem estratégias preventivas eficientes ao desenvolvimento de ações estratégicas que visem à promoção da saúde no alcance da qualidade de vida individual e coletiva. ${ }^{3}$

As síndromes coronarianas agudas são, em geral, causadas por obstrução coronariana decorrente da interação entre fenômenos de trombose e vasoespasmo sobre as lesões ateroscleróticas nas coronárias, podendo manifestar-se com quadros clínicos diversos, tais como: angina instável, infarto agudo do miocárdio (IAM) ou morte súbita. Inicialmente, apenas as placas de ateroma, com obstrução significativa e restrição de luz do vaso em mais de $50 \%$ apresentavam potencial para ruptura, trombose subsequente e oclusão vascular. Porém, estudos recentes desafiam este paradigma e mostram que mesmo 


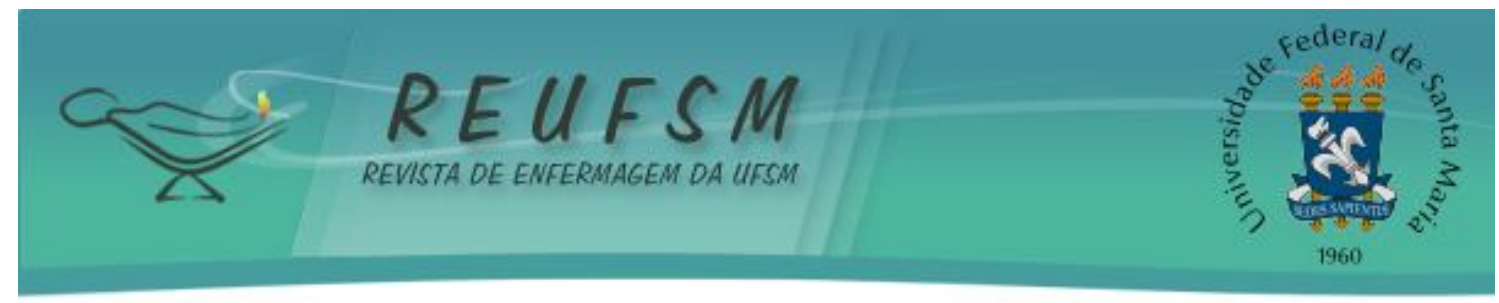

as placas sem lesões obstrutivas significativas, ou seja, com estreitamento da luz vascular menor que $50 \%$ também apresentam potencial trombogênico. ${ }^{4}$

Além disso, a DAC está associada às diversas comorbidades: internações hospitalares; procedimentos diagnósticos e terapêuticos, e tratamento farmacológico e não farmacológico contínuo. De fato, esses fatores determinam um impacto econômico expressivo, pois as estimativas dos custos desses eventos são essenciais para estudos de avaliação econômica e custo-efetividade das tecnologias direcionadas ao manejo da cardiopatia isquêmica. ${ }^{5}$

Tendo em vista as necessidades prementes de se preservar a saúde, a partir do conhecimento sobre as DCV e seus FR, a relevância deste estudo tem o foco no emponderamento do sujeito e dos profissionais de saúde, destacando entre eles: o enfermeiro do trabal ho e da atenção básica. Acredita-se que esses profissionais, na medida em que têm recursos para conhecer o grau de compreensão dos usuários, poderão, de maneira conjunta, traçar metas de prevenção eficazes na perspectiva da coresponsabilidade.

A escolha dos trabalhadores do ramo metal-mecânico para compor a amostra do estudo ocorreu pela característica sócio-econômica do município e pelo fato de que, em algumas indústrias, tem-se o enfermeiro do trabalho, e em outras situações, o enfermeiro da atenção básica assume o papel de educadorassistencial. Muitas vezes, esses trabalhadores são expostos aos FR sem ao menos ter conhecimento, ou não têm a oportunidade de avaliar a sua saúde, por não ter a unidade de saúde aberta em consonância com seus horários, e tampouco aderem às orientações por carência de acompanhamento da situação de saúde atual. Nesse sentido, torna-se relevante que os profissionais atuantes em saúde ocupacional estejam imbuídos de conhecimentos, para que, de fato, possam intervir no processo saúde-doença do trabalhador e da família.

No entanto, observa-se, em consultas de enfermagem, que o usuário busca 0 conhecimento por meio de orientações com profissionais de saúde, material publicado (revistas e livros) ou por meios digitais mas, para muitos deles, em acompanhamento clínico, não se refletem em mudanças de hábitos de vida. Contrapondo, diversos estudos apontam que a prevenção possui um papel fundamental na modificação do estilo de vida que se torna indispensável para a manutenção ou reabilitação da saúde diante do impacto econômico da sociedade e da sobrevida do usuário/trabalhador ${ }^{6-7}$. Face ao exposto, delineou-se este estudo, com o objetivo de identificar o conhecimento dos trabalhadores da indústria metal-mecânica em relação aos fatores de risco para doença arterial coronariana (DAC) e as atitudes voltadas a sua prevenção.

\section{MATERIAIS E MÉTODOS}

Estudo com delineamento transversal, desenvolvido em uma Clínica de Medicina do Trabalho de Caxias do Sul, município da Região Nordeste do Rio Grande do Sul (RS)Brasil, considerado polo metal-mecânico do estado. Esse serviço atende profissionais de diversas áreas para realização de consulta médica, de acordo com o enfoque da procura do trabalhador. As modalidades de atenção constituem-se de exame médico periódico, exame admissional e exame demissional. A clínica conta com 0 atendimento médico, fonoaudiológico e de enfermagem.

A população em estudo são trabalhadores da indústria metal-mecânica que foram atendidos ambulatorialmente na referida instituição. 0 número de atendimentos é em torno de 300 por dia. Os trabalhadores entrevistados foram abordados enquanto aguardavam para realizar a consulta com o profissional de saúde e, após aceitar participar 


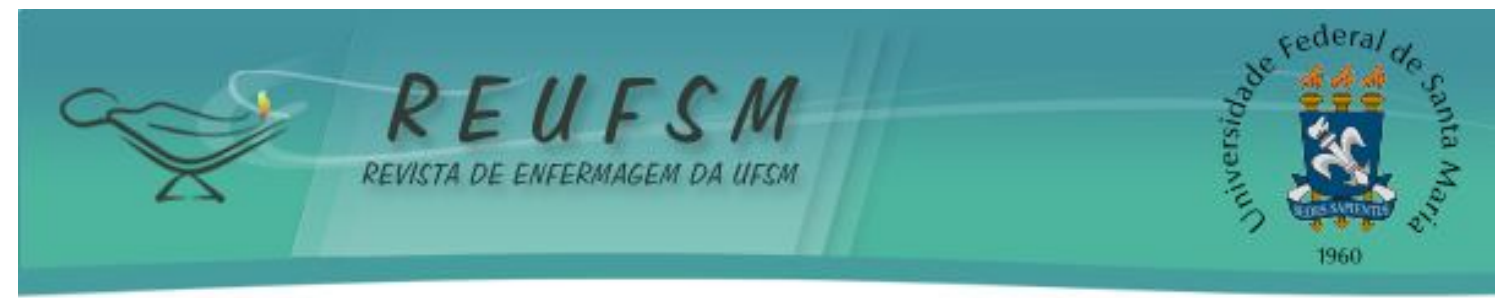

do estudo, foram convidados a se deslocar até um consultório para a realização da entrevista.

A amostra foi do tipo não-probabilística, ou seja, por conveniência, constituída de trabalhadores que atenderam aos critérios de inclusão no estudo: maiores de 18 anos, ambos os sexos, e trabalhar na indústria metal-mecânica. Foram excluídos os trabalhadores com barreiras de comunicação como portadores de deficiência auditiva e de fala ou que não aceitassem participar deste estudo. A coleta de dados foi realizada no mês de abril de 2010.

Os dados foram coletados por meio de um questionário com perguntas fechadas, as quais estavam relacionadas ao conhecimento dos trabalhadores sobre os FR para DAC e as medidas de prevenção. O questionário foi elaborado com base em estudo realizado previamente $^{8}$ e em consonância com a literatura pertinente ao tema proposto. A definição de certo ou errado para cada resposta baseou-se em artigos de revisão de literatura sobre a associação de cada fator de risco e as apresentações clínicas da doença arterial coronariana. $^{9}$

O questionário foi aplicado nos turnos da manhã e tarde, no sentido de abordar grande parte dos trabalhadores. O tempo de duração entre a abordagem dos sujeitos e a realização do questionário foi de, aproximadamente, 15 minutos, e em seguida foram verificados as medidas antropométricas, a pressão arterial (aparelho esfigmomanômetro da marca Sankey e estetoscópio do modelo Rapaporte, ambos com o registro do INMETRO) com o voluntário sentado, após 5 minutos de repouso ${ }^{10}$, a circunferência abdominal verificada na metade da distância entre a crista ilíaca e o rebordo costal inferior (considerada aumentada $>102 \mathrm{~cm}$ para homens e $>88 \mathrm{~cm}$ para mulheres). ${ }^{11} 0$ índice de massa corporal (IMC) foi calculado pela divisão do peso pelo quadrado da altura (metros): baixo peso $\left(<18,5 \mathrm{Kg} / \mathrm{m}^{2}\right)$, eutrófico $\left(18,5-24,9 \mathrm{Kg} / \mathrm{m}^{2}\right)$, sobrepeso $\left(25,0-29,9 \mathrm{Kg} / \mathrm{m}^{2}\right)$, obesidade $\left(30,0-39,9 \mathrm{Kg} / \mathrm{m}^{2}\right) .{ }^{10}$

Caracterizaram-se como tabagistas os trabalhadores que mantinham 0 hábito de fumar cigarros, independentemente da quantidade, e como ex-fumantes os que declararam ter cessado o hábito há pelo menos 30 dias. Considerou-se atividade física o trabalhador que exercia alguma atividade por pelo menos 30 minutos, no mínimo três vezes por semana. A hipercolesterolemia e o diabetes melittus foram auto-referidos, considerados positivos quando em uso de medicação apropriada ou relato de confirmação do diagnóstico por médico.

Para análise dos dados coletados, foi utilizada a estatística descritiva. Após a análise, os dados foram incluídos inicialmente em planilhas do Excel e analisados no programa estatístico SPSS 16.0 para Windows. Os dados foram apresentados em tabelas que descrevem as frequências absolutas, relativas, médias e desvio padrão. Este projeto foi aprovado pelo Comitê de Ética em Pesquisa da Instituição de Ensino Superior de origem, sob o número 067/09 em seus aspectos éticos e metodológicos. Os participantes da pesquisa receberam e assinaram o termo de consentimento livre e esclarecido.

\section{RESULTADOS}

Foram incluídos 100 trabalhadores do ramo metal-mecânico, em que a média de idade foi de $29,0 \pm 10,16$ anos, renda média de $2,0 \pm 1,28$ salários mínimos, sendo a maioria do sexo masculino (66\%). Estes e outros dados estão demonstrados na Tabela 1. 


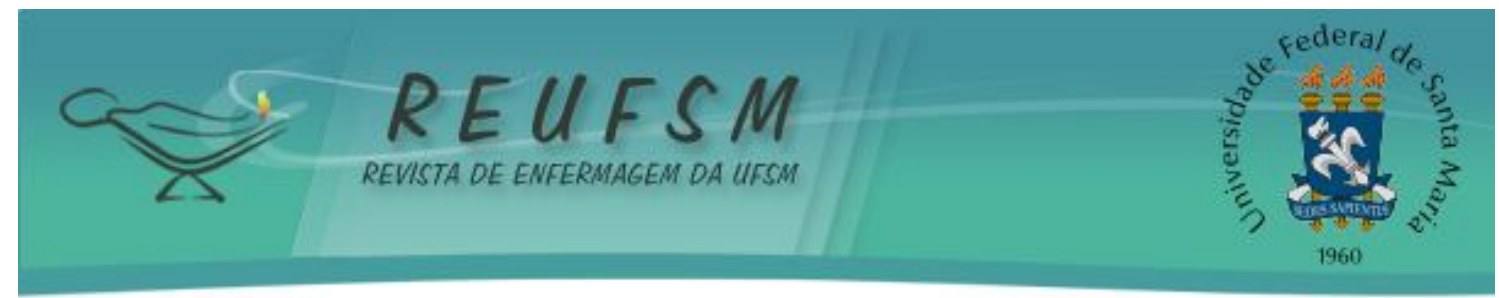

Tabela 1: Características sócio-demográficas. Caxias do Sul- RS, 2010.

*Dados apresentados com média e desvio padrão.

\begin{tabular}{lc}
\hline Variáveis & $\%$ \\
\hline Idade* & $29,0 \pm 10,16$ \\
Sexo masculino & 66 \\
Renda (em salários mínimos)* & $2,0 \pm 1,28$ \\
Escolaridade & \\
$\quad$ Analfabeto & 1 \\
Ensino fundamental incompleto & 10 \\
Ensino fundamental & 41 \\
Ensino médio & 44 \\
Ensino superior & 4 \\
Situação Conjugal & \\
Casado & 40 \\
Solteiro & 47 \\
União estável & 13 \\
Função & \\
Auxiliar de produção & \\
Operador de máquina & 42 \\
Montador & 8 \\
\hline
\end{tabular}

Em relação às variáveis clínicas presentes na amostra, 57\% da amostra relatou ter histórico familiar positivo para DAC, $24 \%$ eram fumantes e $14 \%$ dos trabalhadores referiram ter níveis elevados de colesterol. Nenhum trabalhador referiu história de infarto agudo, e nem ter sido submetido à cirurgia de revascularização do miocárdio. Apenas um trabalhador relatou ter realizado angioplastia (revascularização percutânea). Dados apresentados na Tabela 2.

Tabela 2: Fatores de risco para DAC em trabalhadores. Caxias do Sul- RS, 2010.

\begin{tabular}{lc}
\hline Variá́veis & $\%$ \\
\hline Histórico familiar para DAC & 57 \\
Tabagismo & 24 \\
Fumante & 14 \\
Ex-tabagista & 14 \\
Hipercolesterolemia & 10 \\
HAS & $120,0 \pm 14,7$ \\
Pressão Arterial Sistólica* & $80,0 \pm 12,2$ \\
Pressão Arterial Diastólica* & $24,16 \pm 3,97$ \\
IMC* & 9 \\
DPOC & 3 \\
Diabetes Mellitus & 1 \\
Revascularização percutânea prévia & \\
\hline
\end{tabular}




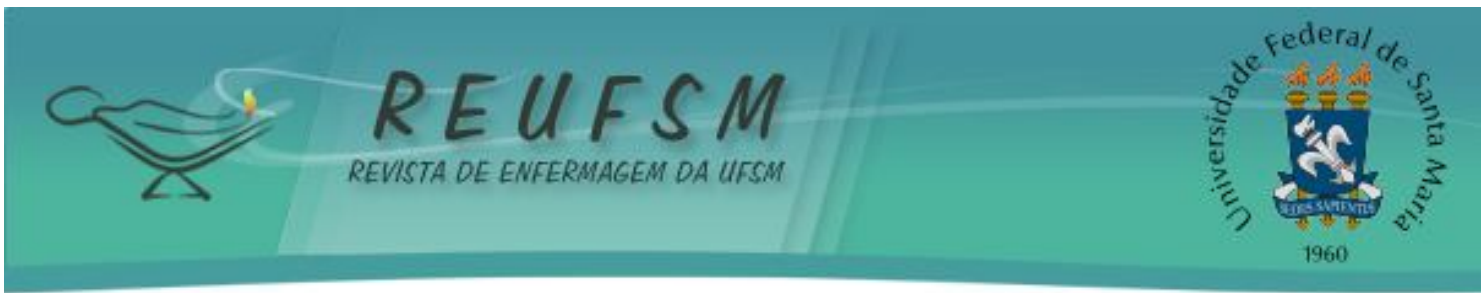

*Dados apresentados com média e desvio padrão. IMC: índice de massa corporal. DAC: doença arterial coronariana, DPOC: doença pulmonar obstrutiva crônica, HAS: hipertensão arterial sistêmica.

\section{Conhecimento sobre fatores de risco para DAC}

A maioria dos trabalhadores (94\%) acredita que a obesidade é um fator de risco para DAC. Este e outros resultados estão apresentados na Figura 1.

Figura 1. Conhecimento sobre os fatores de risco para a DAC. Caxias do Sul - RS, 2010. HAS: hipertensão arterial sistêmica, HFt: história familiar positiva para doença arterial coronariana, DM: diabetes melittus, CA: circunferência abdominal.

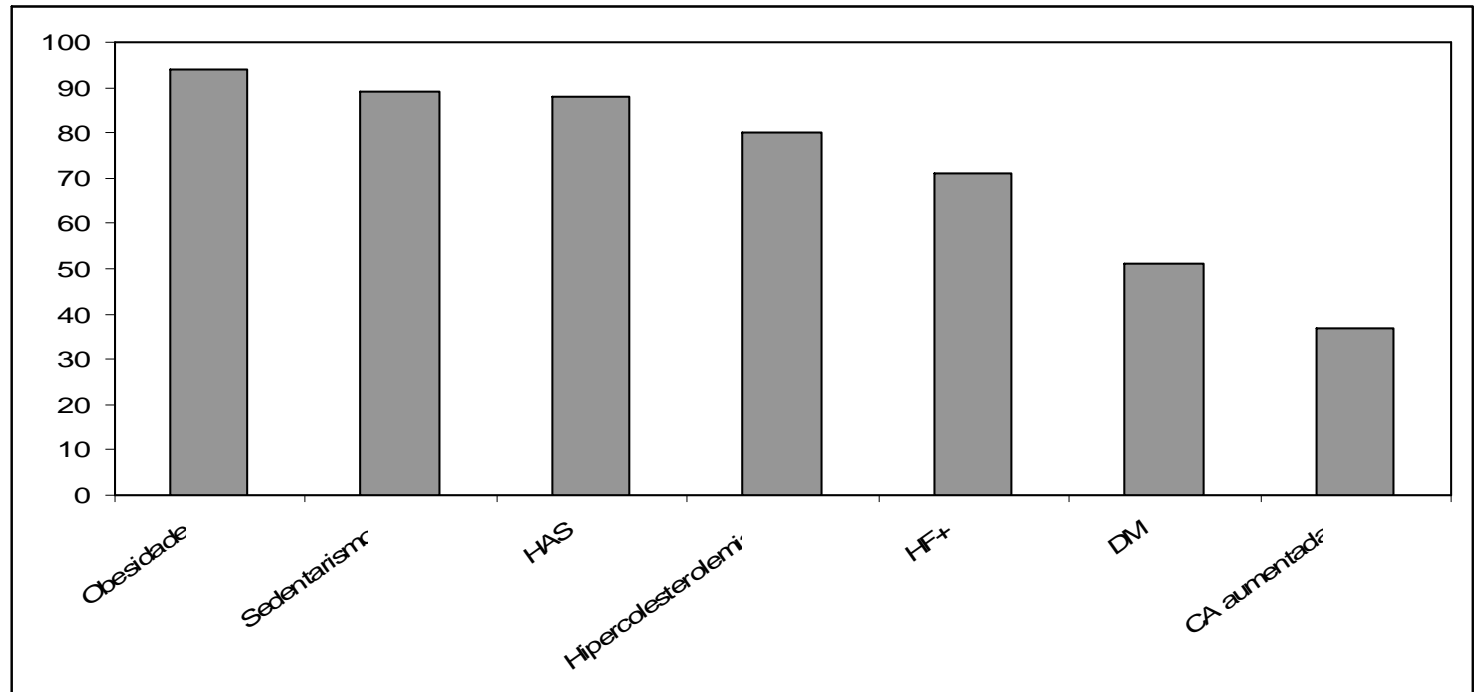

No que se refere ao sexo, 51\% da amostra acreditam que este fator não constitui um fator de risco, ou seja, é indiferente. Já a alimentação gordurosa foi mencionada por $87 \%$ dos entrevistados como sendo um importante $\mathrm{FR}$. Em relação à forma como foram adquiridos estes conhecimentos acerca da DAC, apenas $9 \%$ relataram ler periódicos ou livros sobre o assunto e $5 \%$ referiram esclarecer suas dúvidas na internet. Parte dos entrevistados respondeu obter o que sabem sobre a temática em questão, pelos meios de comunicação como a tel evisão (59\%) e por vizinhos ou amigos (41\%).

\section{Atitudes para prevenção da DAC}

No que se refere às questões relacionadas às atitudes para prevenir a DAC, 51\% dos trabalhadores referiram praticar atividade física pelo menos três vezes por semana. Já em relação ao controle da dieta, somente $22 \%$ da amostra fazem algum controle, como restringir alimentos gordurosos e aumento da ingestão de vegetais (frutas, verduras e legumes). Do total de fumantes, $58 \%$ referiram já ter tentado parar de fumar. Ao serem questionados sobre o controle da pressão arterial (PA), do total dos hipertensos, apenas $40 \%$ deles referiam realizar o controle periódico da PA. Quanto à adesão à terapêutica farmacológica prescrita para as doenças crônicas, do total de trabalhadores que tomavam medicação de uso contínuo, $69 \%$ utilizavam as medicações conforme a prescrição médica. Quanto à avaliação médica periódica, $71 \%$ dos trabalhadores responderam frequentar 0 médico pelo menos uma vez ao ano. 


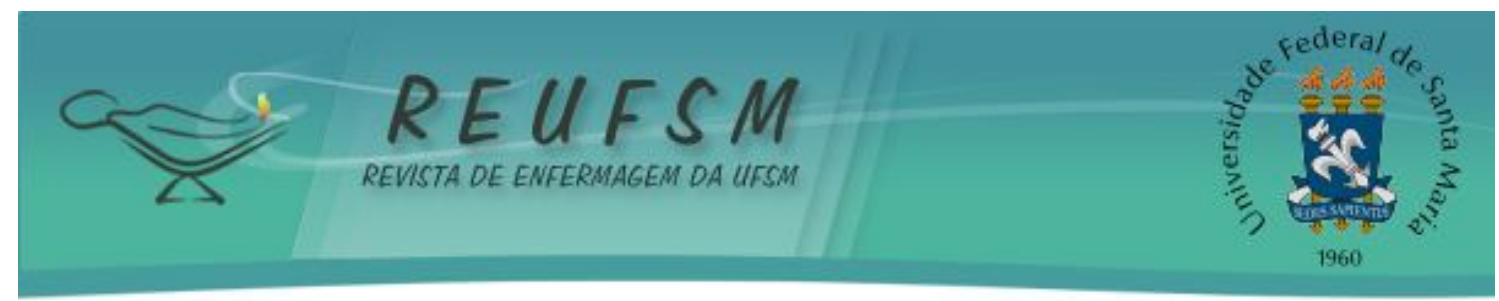

DISCUSSÃO

As características sócio-demográficas, renda familiar e nível de escolaridade vêm sendo cada vez mais relacionadas ao desenvolvimento da DAC. Os fatores de risco tendem a ocorrer com maior frequência e maior número em populações com menor poder econômico e sócio-cultural. Um estudo caso-controle, realizado com 1279 pares de indivíduos, em 51 cidades brasileiras, evidenciou que renda e escolaridade elevadas conferiram proteção contra o infarto agudo do miocárdio (OR 0,$68 ; p=0,02)^{12}$. Conforme os achados deste estudo, $41 \%$ dos trabalhadores tinham completado apenas 0 ensino fundamental completo. A renda média dos trabalhadores era em torno de 2 salários mínimos. Dados recentes de um estudo americano demonstraram que a presença de dois ou mais fatores de risco são mais frequentes entre aqueles com baixa escolaridade (53\%), em comparação com aqueles com curso superior (26\%). ${ }^{13}$

Os estudos de coorte de Framingham também revelam e confirmam a importância de FR para a DAC como: tabagismo, níveis elevados de colesterol LDL, baixos de HDL, diabetes melito, hipertensão arterial sistêmica, história familiar, obesidade, sedentarismo e obesidade central. ${ }^{14}$

O estudo INTERHEART ${ }^{15}$, um caso-controle internacional, avaliou nove fatores de risco que explicou mais de $90 \%$ do risco atribuível, ou seja, da proporção de casos devido ao fator de risco, para infarto agudo do miocárdio (IAM). De forma surpreendente, o tabagismo e a dislipidemia compreenderam mais de dois terços deste risco. Na amostra coletada, $24 \%$ dos trabalhadores referiam ser fumantes. No entanto, $75 \%$ dos trabalhadores afirmaram ter conhecimento de que o tabagismo agrava a DAC, e 58\% do total de fumantes referiram ter tentado parar de fumar alguma vez. O tabagismo é um dos mais importantes fatores de risco para DAC e também para neoplasias, bronquites e enfisema, também considerado pela Organização Mundial de Saúde (OMS) como o mais importante FR evitável de morbimortalidade. A intervenção sobre 0 tabagismo, além de prevenir 0 desenvolvimento da DAC, permite importante redução de morbimortalidade, determinando melhor e maior sobrevida dos indivíduos que param de fumar, de ambos os sexos, e em qualquer faixa etária. ${ }^{13}$

Quanto ao IMC, a média dos trabalhadores atingiu 24,16, ou seja, eram eutróficos. Em nosso estudo, $94 \%$ dos trabalhadores mostraram saber que a obesidade representa um FR para a DAC. No entanto, observou-se que, quando questionados se a circunferência abdominal pode ser um fator de risco, apenas 37\% deles responderam afirmativamente. A hipertensão, na opinião dos trabalhadores (88\%), foi considerada um fator importante de risco para DAC. Todavia, conforme os dados coletados na pesquisa, apenas 10 trabalhadores afirmaram ser hipertensos e, destes, apenas 4 referiam fazer controle da PA.

A maioria $(71 \%)$ da amostra revelou que se submete a algum tipo de avaliação médica, pelo menos, uma vez ao ano. Sabe-se que HAS é um dos mais importantes FR para DAC, mas também aquele de mais fácil abordagem, porque sua prevalência na população adulta é de cerca de $20 \%$ sendo que a detecção e o melhor controle da hipertensão arterial se devem também ao auxílio de exames periódicos, diminuindo a pressão sistólica média desses indivíduos e reduzindo a sua prevalência. ${ }^{13}$

Quando questionados sobre as dislipidemias, $80 \%$ da amostra referiram estar cientes de que o "colesterol alto" é um fator de risco para a DAC. Além da importância da avaliação dos níveis séricos lipídicos para determinação da DAC, faz-se necessária a intervenção sobre essas variáveis lipídicas, com sua modificação nas populações ou em indivíduos portadores de dislipidemias, o que tem sido eficaz na prevenção primária e secundária da doença coronária. ${ }^{16}$ Dados anatomopatológicos e de investigação experimental clínica e epidemiológica, demonstraram que a redução da 


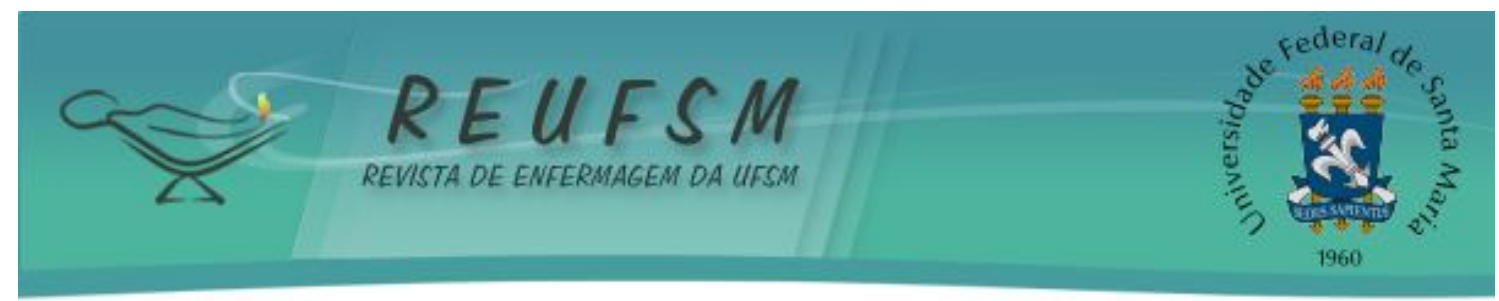

hipercolesterolemia é capaz de diminuir significativamente os eventos clínicos e as mortes decorrentes de DAC, assim como a mortalidade cardiovascular. ${ }^{13}$

0 diabetes parece ter relação com a DAC para a metade dos trabalhadores entrevistados, pois $51 \%$ relataram como sendo um fator de risco. 0 diabetes melitus é um dos maiores fatores de risco para DAC, e cada vez mais, os dados sugerem que muitos desses riscos podem ser minimizados por meio de controle intensivo. Para tanto, as equipes de saúde ocupacional precisam desenvolver atividades de cunho educativo para reforçar a observância de consumo diário e informações sobre os alimentos que devem ser evitados, bem como a utilização de adoçantes à base de aspartame. ${ }^{17}$

Outro FR que merece destaque é o sedentarismo. A falta de atividade física é hoje um dos grandes vilões da DAC. O exercício físico regular tem efeitos favoráveis sobre os fatores de risco, incluindo a normalidade nos parâmetros referentes aos níveis de insulina, glicose, coagulação e fibrinólise. ${ }^{3}$ Felizmente, metade da amostra, ou seja, 51\% afirmaram exercer alguma atividade física pelo menos três vezes por semana, por no mínimo trinta minutos, incluindo caminhadas, corrida, academia, entre outros. O sedentarismo também foi citado por $89 \%$ dos trabal hadores como sendo FR para coronariopatia.

Com relação ao histórico familiar como fator de risco, 71\% mostraram estar cientes de que ele representa um fator de risco importante para doença, sendo que $57 \%$ da amostra referiram ter algum familiar em primeiro grau com doença cardíaca. Portanto, 0 fato de se ter algum familiar com doença cardíaca atribui a homens e mulheres um risco aumentado para DAC, provavelmente, por uma combinação de fatores genéticos e ambientais, considerado como herança multifatorial. ${ }^{3} \mathrm{~A}$ diferença entre homens e mulheres com relação à DAC ainda não é bem clara para a maioria dos trabalhadores, como foi constatado nesta pesquisa, apenas $49 \%$ deles acreditam que os homens sofrem mais de DAC do que as mulheres. Porém, sabe-se que é conferida à mulher uma proteção extra aos danos cardiovasculares em decorrência da ação de hormônios como o estrogênio, por exemplo. ${ }^{3}$

Os dados da mortalidade brasileira² por DAC apontam a prevalência para homens; entretanto, no município em que foi realizado esse estudo, a mortalidade das mulheres é superior. Nesse sentido, observa a necessidade de estudos para esclarecer os riscos relacionados ao sexo. Contudo, sabe-se que a menopausa em idade mais precoce aumenta o risco de DAC, independentemente do mecanismo de menopausa. Entre mulheres que se submeteram à ooforectomia bilateral, por exemplo, aquelas com menos de 35 anos tem quase oito vezes o risco para IAM não fatal comparado com a menopausa natural. ${ }^{3}$

A maioria das informações adquiridas pela amostra em estudo, segundo eles, foi pela TV, seguido por opiniões e conversas com vizinhos, amigos e colegas de trabalho, além de ter alguém com doenças cardiovasculares na família. O esclarecimento sobre os FR para DAC e outras doenças cardiovasculares, em geral, assume um papel fundamental de prevenção. Por estes fatores, tem-se evidenciado cada vez mais o papel do enfermeiro como educador em saúde, atuando na atenção primária, assim como na medicina preventiva, com visão no futuro, realizando, por exemplo, grupos de apoio para hipertensos, diabéticos, obesos, buscando com isto a prevenção de doenças e uma melhor qualidade de vida às pessoas deste grupo específico, ou seja, da indústria metal-mecânica.

Todavia, tornam-se relevantes esses achados, pois corroboram a necessidade de modificação dos hábitos de vida para controle dos fatores modificáveis associados, contribuindo para a redução dos riscos associados para a DAC. ${ }^{18}$ Portanto, esses resultados podem ser utilizados para elaborar estratégias de atenção à saúde do trabalhador, visando o envelhecimento ativo e saudável sob a perspectiva da promoção da saúde, tendo em vista a plena capacidade para o trabalho. A redução de agravos de longa duração e de 


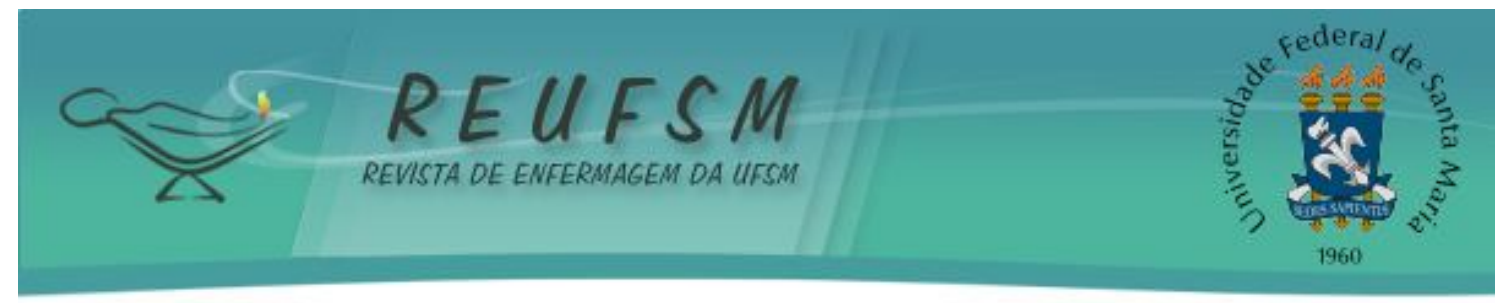

absenteísmo do trabalho possibilita à indústria maior produtividade e lucro, assim como eliminar custos desnecessários relacionados à previdência social. Deste modo, recomendam-se também novos estudos que conheçam a capacidade para o trabalho de outros setores e segmentos da indústria para que, dessa maneira, os trabalhadores sejam acompanhados durante sua traj etória laboral.

\section{CONCLUSÕES}

A obesidade foi considerada como fator de risco para DAC pela maioria dos trabalhadores, seguida pela hipertensão arterial e hipercolesterolemia. Em relação às atitudes para prevenção da DAC, metade da amostra referiu praticar atividade física.

Face aos resultados, observa-se a necessidade de um esclarecimento maior dos profissionais de saúde para exercer o pleno papel como educador em saúde, em decorrência das dúvidas identificadas com relação à DAC, bem como dos FR. A amostra incluída representa uma parcela dos trabalhadores da indústria metal-mecânica, a qual está constantemente exposta aos riscos ocupacionais e fatores de risco que podem contribuir significativamente para a incidência da DAC.

Salienta-se que as DCV não vêm sendo tratadas com a devida importância pelas indústrias, como possível causa de afastamentos, invalidez e até mesmo o óbito prematuro de seus trabalhadores. A detecção dos FR frequentes e modificáveis, assim como a identificação do risco cardiovascular e a intervenção educativa para desenvolver atitudes e comportamentos saudáveis podem contribuir para a redução da morbimortalidade, que são potencialmente causadoras de deficiências e incapacidades para o trabalho.

A saúde ocupacional com a atuação do enfermeiro do trabalho vem adquirindo um papel cada vez mais importante na prevenção de doenças relacionadas ao trabalho no mercado atual, pois exige profissionais de saúde habilitados para o exercício da função, de maneira que garanta ao trabalhador a prevenção de doenças e a promoção da saúde, no sentido do alcance de uma qualidade de vida individual e coletiva, assim como na perspectiva de um envel hecimento ativo. Nesta seara, a contribuição deste estudo para a enfermagem do trabalho compreende em conhecer 0 grau de entendimento dos trabalhadores das indústrias que estão expostos aos diversos riscos dos agravos de longa duração, de modo que possam ser instituídos planos de ação conjunta com os próprios trabalhadores voltados à prevenção primária, no sentido de prolongar a vida com qualidade, por meio da proteção, da promoção e do restabelecimento da saúde.

Entretanto, a saúde do trabalhador requer ir mais além do que a legislação vigente que traz normas e condutas a serem seguidas pelas indústrias. Deve-se exigir que se cumpra a parte legal por meio dos levantamentos de riscos ambientais e dos programas de saúde ocupacional, visando à prevenção de acidentes e de doenças ocupacionais, inclusive de agravos de longa duração. Estas ações também ficam a cargo do médico e do enfermeiro do trabalho, sendo que este último tem responsabilidade ainda maior no que diz respeito à prevenção a partir da educação em saúde, a qual precisa ser co-planejada e co-responsabilizada com o trabal hador.

$\mathrm{Na}$ perspectiva da redução dos riscos para as DAC, veem-se necessários novos estudos que identifiquem associações entre as condições de trabalho e os fatores de risco para a DAC, pois com o aumento de carga de trabalho, as cobranças/ exigências, a necessidade de tomada de decisão e a responsabilidade do cargo podem denotar a redução da capacidade vital para o trabalho. Torna-se necessário o acompanhamento das condições de saúde, de modo que possa intensificar as ações de promoção da saúde de forma sistemática e extensiva para todos os trabalhadores. 


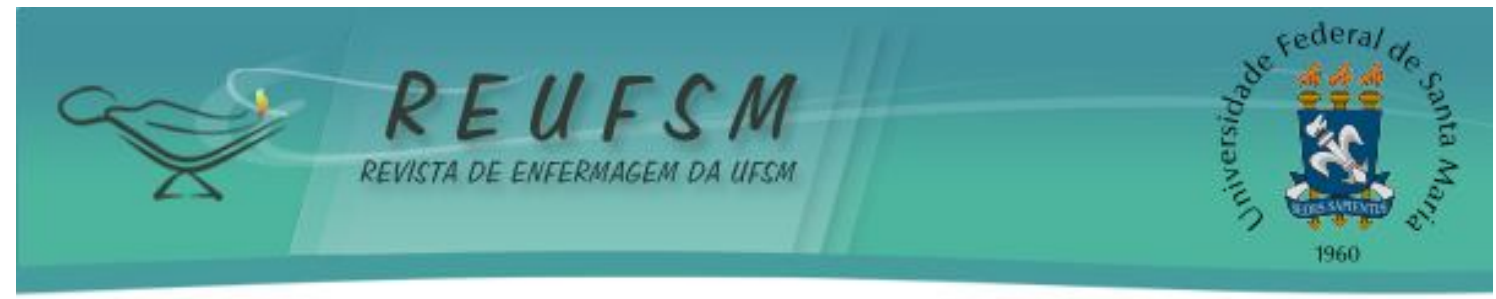

\section{REFERÊNCIAS}

1. Stipp M, Cunha N. Cardiovascular risk in ambulatorial clientele - a quantitative study. Online Brazilian J ournal of Nursing [periódico na internet]. 2007 November 18; [acesso em 02 fev. 2011]; 7(0):[aproximadamente 6 p.]. Disponível em: http:/ / www. objnursing. uff. br/ index. php/ nursing/ article/ view/ 1213

2. Ministério da Saúde (BR). Departamento de Informática do Sistema Único de Saúde. Estatísticas vitais: mortalidade. Brasília ; 2009 [acesso em 22 ago. 2010]. Disponível em: http:/ / tabnet. datasus. gov. br/ cgi/ tabcgi. exe?simp/ cnv/ obtrs. def.

3. Woods SL, Froelicher ESS, Motzer SU. Enfermagem em cardiologia. 4ạ ed. São Paulo: Manole; 2005.

4. Pinheiro MGV, Rabelo Jr AR, Jesus RS, Nascimento LC, Costa UMM. Síndromes coronarianas agudas na ausência de doença arterial coronariana significativa. Arq Bras Cardiol. 2005; 84(1):24-8.

5. Ribeiro RA, Mello RGB, Melchior R, Dill J C, Hohmann CB, Lucchese AM, et al. Custo anual do manejo da cardiopatia isquêmica crônica no Brasil: perspectiva pública e privada. Arq Bras Cardiol. 2005; 85(1):3-8.

6. Araújo DV, Ferraz MB. Impacto econômico do tratamento da cardiopatia isquêmica crônica no Brasil. O desafio da incorporação de novas tecnologias cardiovasculares. Arq Bras Cardiol [periódico na Internet]. 2005 [acesso em 15 ago. 2010]; 85(1):1-2. Disponível em: http:// www. scielo.br/pdf/abc/ v85n1/a01v85n1.pdf

7. Pinheiro DGM, Pinheiro CHJ, Marinho MJ F. Estilo de vida e importância da educação em saúde na reabilitação cardíaca após cirurgia de revascularização do miocárdio. Rev Bras Prom Saúde [periódico na Internet]. 2007 [acesso em 15 ago. 2010]; 20(4):213-20. Disponível em: http:/ / redalyc. uaemex. mx/ redalyc/ html/ 408/ 40820403/ 40820403. html

8. Borges TT, Rombaldi AJ, Knuth AG, Hallal PC. Conhecimento sobre fatores de risco para doenças crônicas: estudo de base populacional. Cad Saúde Pública. 2009; 25(7):1511-20.

9. American Heart Association/ American College of Cardiology (AHA/ ACC). Guidelines for Secondary Prevention for Patients With Coronary and Other Atherosclerotic Vascular Disease: 2006 Update. Circulation. 2006; 113:2363-72.

10. Sociedade Brasileira de Cardiologia. I Diretriz Brasileira de Diagnóstico e Tratamento da Síndrome Metabólica. Arq Bras Cardiol. 2005; 84(supl I):1-28.

11. Lunelli RP, Portal VL, Esmerio FG, Moraes MA, Souza EN. Adesão medicamentosa e não medicamentosa de pacientes com doença arterial coronariana. Acta paul enferm [periódico na internet]. 2009 [acesso em 15 ago. 2010]; 22(4): 367-3. Disponível em: http:/ / www. scielo.br/ pdf/ ape/ v22n4/ a03v22n4.pdf

12. Piegas LS, Avezum A, Pereira J C, Rossi Neto JM, Hoepfner C, Farran J Á, et al. Risk factors for myocardial infarction in Brazil. Am Heart J. 2003; 146(2): 331-8.

13. Ciorlia SLA, Godoy MF. Fatores de risco cardiovascular e mortalidade. Seguimento em longo prazo (até 20 anos) em programa preventivo realizado pela medicina ocupacional. Arq Bras Cardiol. 2005; 84(3):20-5.

14. Wilson PW, Agostino RB, Levy D, Belanger AM, Silbershatz H, Kannel WB. Prediction of coronary heart disease using risk factor categories. Circulation. 1998; 97:1837-47. 


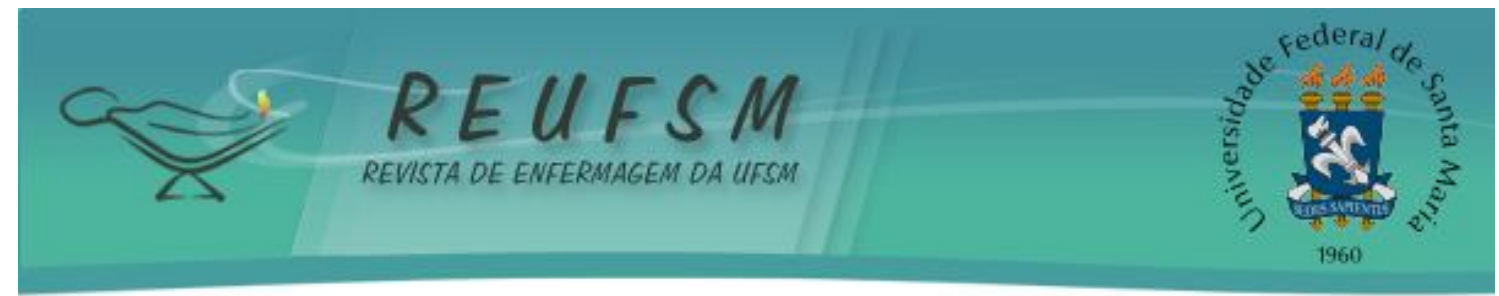

15. Polanczyk CA. Fatores de risco cardiovascular no Brasil: os próximos 50 anos! Arq Bras Cardiol. 2005; 84(3):199-201.

16. Bertolami MC, Faludi AA, Latorre MRDO, Zaidan Filho T. Perfil lipídico de funcionários de indústria metalúrgica e sua relação com outros fatores de risco. Arq Bras Cardiol. 1993; 60(5):293-9.

17. Souza J A, França ISX. Prevalência de hipertensão arterial em pessoas com mobilidade física prej udicada: implicações para a enfermagem. Rev Bras Enferm. 2008; 61(6):816-21.

18. Alves A, Marques IR. Fatores relacionados ao risco de doença arterial coronariana entre estudantes de enfermagem. Rev Bras Enferm. 2009; 62(6):883-8.

Data de recebimento: 15/02/ 2011

Data de aceite: 01/04/2011

Contato com autora responsável: Emiliane Nogueira de Souza

Rua: Sarmento Leite, 245 / Sala 411 Porto Alegre - RS CEP: 90050-170

E-mail: emilianes@ufcspa.edu.br 